\title{
Desde la areografía de Rapoport a los 30 años de la macroecología: Síntesis de la contribución argentina
}

\author{
Paula N. Fergnani ${ }^{1} \&$ Julieta Filloy ${ }^{2}$ 凹 \\ ${ }^{1}$ Laboratorio Ecotono. INIBIOMA-UNCOMA, CONICET. ${ }^{2}$ Laboratorio de Ecología de Comunidades y Macroecología. EGE- \\ IEGEBA, UBA-CONICET.
}

\begin{abstract}
Resumen. Desde que apareció el libro Areografía y se consolidó la macroecología en la década del ochenta, la investigación centrada en las leyes generales que gobiernan la distribución de los organismos en el planeta creció de forma marcada. Eduardo Rapoport dejó un legado en la Argentina y en el mundo. Nos preguntamos en qué medida los ecólogos argentinos incorporamos el enfoque macroecológico e interactuamos con investigadores extranjeros, cómo es la inserción internacional y en qué aspectos nos centramos. Realizamos una búsqueda de artículos en Scopus incluyendo las palabras macroecology, areography, macroecología y areografía, y Argentina en la filiación. Encontramos 253 artículos que muestran que el número de artículos de macroecología publicados por autores argentinos en el tiempo es creciente y con tendencia similar a la global. El número de citas anuales a Areografía se estabilizó en 30 en trabajos internacionales. La mayoría de los autores argentinos publicó dos artículos como máximo, mientras que la minoría publicó entre 3 y 22, lo que sugiere un crecimiento basado en contribuciones eventuales más que en programas de investigación en macroecología. La mayor parte de los artículos publicados por argentinos se centró en el estudio de las áreas de distribución, patrones en los rangos geográficos y en la riqueza de especies. El grupo taxonómico más estudiado fue el de los invertebrados, una característica idiosincrática de la macroecología argentina. En el 70\% de los trabajos, el autor de correspondencia fue argentino, lo cual sugiere un rol protagónico. Las revistas que publicaron con mayor frecuencia los trabajos argentinos coinciden con las que publicaron los trabajos internacionales. La inserción de la Argentina en la macroecología internacional es clara. El establecimiento de programas regulares de investigación con enfoque macroecológico es un área relativamente vacante con muchas posibilidades de expansión.
\end{abstract}

[Palabras clave: biogeografía, Regla de Rapoport, riqueza de especies, corología, revisión bibliométrica, bibliometrix, cienciometría]

Aвstract. From Rapoport's areography to 30 years of macroecology: A synthesis of the Argentinian contribution. Since the appearance of Areography and the consolidation of macroecology in the ' 80 s, research focused on the general laws that govern the distribution of organisms on the planet has increased markedly. Eduardo Rapoport has left a legacy in Argentina and in the world. We ask to what extent Argentinian ecologists incorporate the macroecological approach, interact with foreign researchers, are inserted in the international level and what are the main focuses of their studies. We searched for articles in Scopus containing the words macroecología, areografía, macroecology and areography, and Argentina in the affiliation. We found 253 articles that show that, with a decade of delay, the number of macroecological articles published by Argentinian authors over time increases with a trend similar to the global one. Currently, 30 citations are made to Areography per year in international studies. Most of the Argentinian authors published a maximum of two articles, while the minority published between 3 and 22, suggesting that the increasing trend is based on eventual contributions rather than on research programs on macroecology. Most of the Argentinian articles focused on the study of distribution areas, geographic species ranges and their patterns and species richness. The most studied taxonomic group was that of invertebrates, likely explained by Argentinian macroecology idiosyncrasy. In $70 \%$ of the studies, the correspondence author was Argentinian, suggesting a leading role. The journals in which Argentinian manuscripts were published most frequently matched with those where international papers were published. Argentina is clearly inserted in international macroecology; the establishment of regular programs with a macroecological focus is a relatively vacant area plenty of possibilities for its expansion.

[Keywords: biogeography, Rapoport's Rule, species richness, corology, bibliometric review, scientometrics]

Editora asociada: María Semmartin $\bar{\Delta}$ jfilloy@ege.fcen.uba.ar
Recibido: 14 de Abril de 2020

Aceptado: 25 de Octubre de 2020 


\section{INTRODUCCIÓN}

La investigación de las causas que determinan la abundancia y la distribución de las especies ocupó tanto a los primeros naturalistas como a los científicos desde los comienzos de la ecología como ciencia formal. En su trabajo fundacional, los exploradores Humboldt y Bonpland (1807) realizaron un análisis de la distribución de las especies de plantas en gradientes altitudinales de Ecuador. Al demostrar que ésta se encuentra asociada con gradientes climáticos, esbozaron las primeras hipótesis acerca de los factores que determinan la distribución geográfica de la biodiversidad (Hawkins 2001). Estas hipótesis sentaron las bases conceptuales para el desarrollo de la biogeografía como disciplina científica y el interés por analizar los factores ambientales que promueven el mantenimiento de los patrones geográficos de diversidad biológica (Hoorn et al. 2019; Shrodt et al. 2019).

En el siglo XX se consolidaron las ideas de Humboldt y la ecología geográfica a través de numerosas publicaciones científicas dedicadas al estudio de la variación espacial en la diversidad biológica y sus posibles causas (e.g., MacArthur and Wilson 1967; Brown 1984; O'Brien 1998). Una de las publicaciones más influyentes fue Geographical Ecology, por Robert H. MacArthur (1972), quien definió a la ecología geográfica como una ciencia de patrones. El concepto de patrón o regularidad es central para la ciencia. El patrón implica algún tipo de repetición, y en la naturaleza suele ser una repetición imperfecta, pero que hace posible realizar predicciones y efectuar comparaciones (MacArthur 1972). A partir de patrones en la distribución de plantas $\mathrm{y}$ animales que pueden ser representados en un mapa, MacArthur elaboró conceptos teóricos fundamentales que transformaron la ecología. Entre otros, realizó aportes innovadores al teorizar sobre la distribución especie-abundancia, la distribución de tamaños corporales, la relación especies-área y especies-tiempo y el gradiente latitudinal en el número de especies, conceptos que hoy son universales y que sentaron las bases de la macroecología (Brown 1999).

Con una impronta fuerte de la ecología geográfica de MacArthur y la biogeografía de Humboldt, la macroecología se define a fines de la década del ochenta (Brown and Maurer 1989). En principio, se la definió como el estudio de las relaciones entre organismos y el ambiente, involucrando la caracterización e interpretación de patrones estadísticos en abundancia, distribución y diversidad, a grandes escalas espaciales (geográficas) y temporales (Brown and Maurer 1989; Brown 1995). Para Lawton (1999), la macroecología "es una mezcla entre la ecología, la biogeografía y la evolución, y trata de situarse por encima de detalles [...] para encontrar una imagen más grande..."; "es la búsqueda de patrones estadísticos en los tipos, distribuciones, abundancias y riqueza de especies, desde la escala local a global, y el desarrollo y prueba de explicaciones teóricas subyacentes para esos patrones". La biogeografía, por su parte, se centra en la geografía de la vida (Lomolino et al. 2017). Es el estudio de la variación geográfica en características genéticas, morfológicas, poblacionales y comunitarias, entre otras, a través de la superficie terrestre (Lomolino 2020). La 'mezcla', es decir, el grado de superposición entre la macroecología y la biogeografía, y la consideración de la segunda en la definición de la primera, es sujeto de debate (e.g., Blackburn and Gaston 2002; Fisher 2002; Kent 2005; McGill 2019). Estas discusiones se dieron debido a la coexistencia de distintas visiones sobre la macroecología. Hoy se pueden reconocer dos definiciones conceptuales que resaltan cada una dos aspectos pilares de la macroecología. La macroecología como la ecología de la gran escala (Brown and Maurer 1989) y la macroecología como la identificación de principios generales independientemente de la escala de análisis (Marquet 2002) (Figura 1). Estas definiciones no son mutuamente excluyentes y operan de forma complementaria. Aunque en la práctica muchos trabajos de macroecología son a gran escala geográfica, el consenso es que la macroecología no se limita a esa escala (McGill 2019). En la actualidad, la macroecología se encuentra consolidada dentro de la ecología, y recientemente se celebró su aniversario número 30 (Brown 2019).

En la Argentina, el desarrollo de la ecología desde una perspectiva macro-geográfica se comienza a plasmar en análisis pioneros de patrones de diversidad (e.g., Rabinovich and Rapoport 1975; Rapoport et al. 1976). Simultáneamente, en 1975, el ecólogo argentino Eduardo H. Rapoport (1975) publicó Areografía, y esta obra se convirtió en una pieza fundamental para el desarrollo de la macroecología. En 2020, Areografía cumplió su aniversario número 45. En este libro, Rapoport desarrolla teoría en relación con las estrategias 
geográficas de las especies estudiando sus áreas de distribución geográfica. Rapoport (1975) definió a la areografía, muy relacionada con la corología, como el estudio de la distribución espacial de los taxa (e.g., subespecies, especies, géneros, familias, etc.) con interés en la forma y el tamaño de sus áreas de distribución. Así, se puede considerar que la areografía es una antecesora cercana y muy asociada con la macroecología. De hecho, con una raíz común en la biogeografía y la ecología, la definición conceptual de areografía puede ser incluida dentro de la de macroecología (Figura 1). La primera versión de Areografía (Rapoport 1975) fue publicada por el Fondo de Cultura Económica en México, en español, y su incorporación en el contexto científico mundial se vio demorada. La versión en inglés (Rapoport 1982) cobró notable relevancia en la comunidad científica (Brown 2017), fundamentalmente a partir de la observación de una tendencia de incremento del tamaño de los rangos geográficos de las especies desde los trópicos hacia regiones templado-frías, observado por Rapoport para mamíferos norteamericanos. Este patrón fue rescatado en la literatura posterior como una regla eco-geográfica denominada Regla de
Rapoport (Stevens 1989) (Figura 1), y continúa siendo explorada hasta la actualidad (e.g., Villalobos et al. 2018; Outomuro et al. 2019). De acuerdo con Brown (2017), Areografía es un tesoro; la originalidad de su perspectiva geométrica para entender la distribución de las especies en el espacio propone una fuente de ideas única por redescubrir.

En este trabajo realizamos una síntesis del recorrido argentino en la subdisciplina de la macroecología en base a una revisión bibliográfica. Reconociendo el límite conceptual difuso con la biogeografía, nos centramos en analizar el desarrollo de la macroecología como un marco conceptual que puede, o no, incluir aspectos biogeográficos, pero que es clave para avanzar en los principios generales que rigen los sistemas ecológicos escapando de la contingencia (McGill 2019). La macroecología es una subdisciplina en auge en el mundo (McGill 2019) y en la que se investiga activamente en América Latina (e.g., Rodríguez et al. 2017 [México]; de Moraes Weber 2018 [Brasil]) y en la cual Eduardo Rapoport dejó un legado tanto local (en la Argentina) como mundial (Brown 2017). Sin embargo, no existe ningún estudio

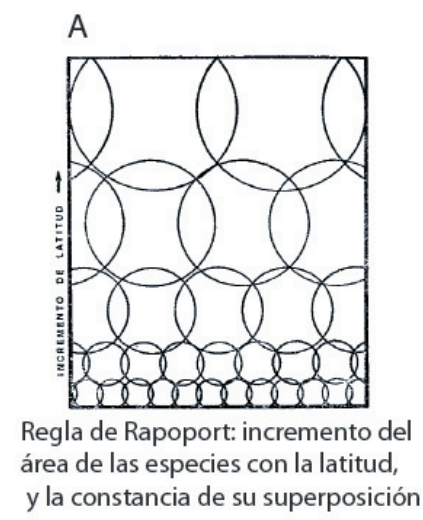

"La areografía (corología) es el estudio de las áreas de distribución de los taxones (subespecies, especies, géneros, familias, etc.)"

“...interesa la forma y el tamaño de las áreas de dispersión de las especies. Es el estudio de la distribución espacial de los taxa, pero a nivel geográfico" Rapoport (1975)

yla constancia de su superposición

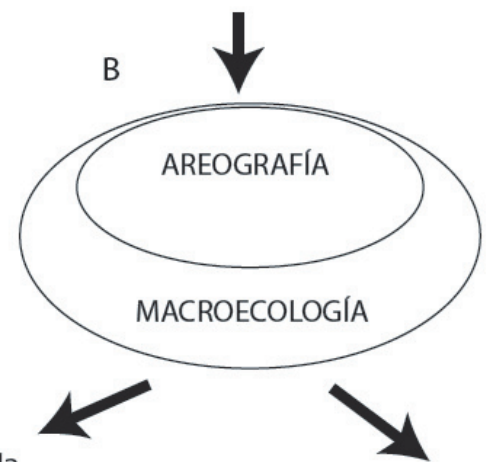

La ecología de la gran escala

"...la división de los alimentos y
el espacio entre las especies en los continentes"
Brown \& Maurer (1989)
"La macroecología tiende a focalizarse en
fenómenos a escalas espaciales desde regional a
global y a escalas temporales desde décadas a
milenios" Brown (1995)

La búsqueda de regularidades

"La macroecología identifica principios generales o reglas naturales que explican la estructura y funcionamiento de los sistemas ecológicos... no importa la escala del análisis" Marquet (2002)

Figura 1. Esquemas conceptuales de: a) la relación entre los términos areografía y macroecología, y b) la Regla de Rapoport (tomada de Rapoport 1975). Las citas son textuales.

Figure 1. Conceptual diagrams of: a) the relationship between the terms areography and macroecology, and b) the Rapoport's Rule (taken from Rapoport 1975). References are textual. 
sobre cómo se desarrolló la macroecología en la Argentina. Así, teniendo en cuenta la historia de la macroecología en general y en la Argentina en particular, nos preguntamos: Los ecólogos argentinos ¿Incorporamos el enfoque macroecológico en nuestros programas de investigación? ¿En qué nos centramos? ¿Existe una preponderancia de estudios de rangos geográficos y áreas de distribución de las especies? ¿La macroecología argentina se encuentra inserta en el ámbito internacional? Los autores argentinos $i$ Tienen un rol central en las publicaciones? Respondimos estas preguntas a partir de artículos seleccionados de la base de datos Scopus. Representamos las publicaciones de interés a través de los artículos que mencionaron las palabras macroecología y areografía, y con al menos un autor con filiación argentina.

\section{Materiales y Métodos}

\section{Recopilación de datos}

Realizamos una búsqueda de artículos publicados hasta abril de 2019, utilizando la base de Scopus. Incluimos aquellos artículos conteniendo Argentina en la filiación y que mencionaron los términos macroecology, areography, macroecología o areografía en el título, en las palabras clave o en el resumen. También incorporamos la palabra aerografía y aerography, dado que encontramos menciones al libro de Rapoport con las letras ' $e$ ' y ' $r$ ' invertidas. Debido a la poca cantidad de artículos resultantes extendimos la búsqueda de esas palabras a todos los campos. Luego repetimos el procedimiento, pero para los artículos publicados a nivel global, independientemente de la filiación. Encontramos que con anterioridad a 1996 existen artículos en Scopus que se encuentran cargados en una versión escaneada y, por lo tanto, las palabras dentro del texto no son reconocibles. Por lo tanto, para el período 1989-1996 buscamos los artículos por nombre del autor considerando a los miembros del grupo de trabajo dirigido por E. Rapoport luego de su llegada al país, para poder precisar el progreso de la macroecología y la areografía en la Argentina. Utilizar Scopus como única fuente bibliográfica puede resultar en la subestimación de la cantidad de artículos publicados en macroecología con al menos un autor argentino; sin embargo, los artículos obtenidos con nuestros criterios de búsqueda y selección son una muestra representativa de los artículos de interés para este estudio.
Los artículos de autores argentinos obtenidos con los criterios de búsqueda mencionados fueron revisados para excluir aquellos que contenían alguno de los términos, pero no correspondían a un estudio ecológico o correspondían a otras disciplinas (e.g., antropología). Una vez seleccionados los artículos con la palabra Argentina, construimos la base de datos identificando el nombre de los autores con filiación argentina (i.e., autores argentinos a partir de aquí), el año de la publicación, el nombre de la revista, la nacionalidad de los coautores, las palabras clave y las referencias bibliográficas. Para caracterizar el área temática abordada por cada trabajo seleccionado identificamos las variables de estudio consideradas y los grupos taxonómicos involucrados en los estudios. Debido a la variabilidad en la terminología para variables y especies o grupos de especies incluidos en cada artículo, éstos fueron clasificados según los términos comúnmente utilizados en la literatura macroecológica (e.g., Rodríguez et al 2017). Las categorías no fueron excluyentes, ya que, por ejemplo, un artículo que estudió varios grupos taxonómicos fue registrado en más de una oportunidad. Entonces, se analizó la frecuencia relativa de aparición de los diferentes grupos taxonómicos y variables estudiadas.

\section{Análisis de datos}

Realizamos un análisis bibliométrico de la base de datos elaborando gráficos descriptivos. Específicamente: 1) realizamos un diagrama de dispersión para examinar la tendencia temporal en el número de artículos publicados en macroecología con autores con filiación argentina (artículos argentinos a partir de aquí) en comparación con la tendencia internacional; 2) estudiamos la distribución de la cantidad de contribuciones entre los autores con filiación argentina graficando la frecuencia de autores en función de la cantidad de artículos argentinos por autor;3) evaluamos en qué medida las palabras macroecología y areografía son utilizadas en los principales criterios de búsqueda bibliográfica y cuáles son las palabras claves más frecuentes mediante una nube de palabras; 4) identificamos los énfasis en las áreas temáticas abordadas a partir de gráficos de frecuencias absolutas de las variables y grupos taxonómicos registrados y comparamos de manera cualitativa sus valores; 5) analizamos la nacionalidad de los coautores en los trabajos argentinos mediante un gráfico de frecuencias, y en qué medida 
los autores argentinos tienen el rol principal en las publicaciones (i.e., son autores de correspondencia); 6) comparamos, mediante un gráfico de frecuencias, las principales revistas en las que los artículos argentinos y extranjeros fueron publicados; y 7) analizamos las 15 publicaciones más citadas en los artículos argentinos. Realizamos los análisis en R project (R Core Team 2019). Utilizamos los paquetes bibliometrix (Aria and Cuccurullo 2017) y wordcloud 2 (Lang and Chien 2018).

\section{Resultados}

Con los criterios de búsqueda detallados previamente encontramos 328 artículos publicados por al menos un autor argentino, de los cuales 253 fueron seleccionados para el análisis por ser estudios relacionados con el enfoque macroecológico y del área de ecología. A su vez, esas palabras se encontraron en
11688 artículos publicados por autores de todas las nacionalidades. La cantidad de publicaciones con al menos un autor argentino a través del tiempo fue creciente (Figura 2A), y la tendencia fue similar a la global iniciándose con media década de retraso (Figura 2B). Existieron unas pocas menciones de la palabra macroecología previas a su definición en 1989, y correspondieron a otros usos de la palabra. El uso del término areografía resultó escaso entre autores argentinos (Figura 2C), y a nivel global se estabilizó en aproximadamente 30 artículos por año (Figura 2D).

Los autores argentinos que publicaron artículos con un enfoque macroecológico fueron en total 594. Cada autor participó en entre 1 y 22 artículos; 535 autores (90\%) publicaron un único artículo, 56 autores $(9.5 \%)$ publicaron entre 2 y 8 artículos y $3(0.5 \%)$ publicaron entre 12 y 22 artículos (Figura 3). El
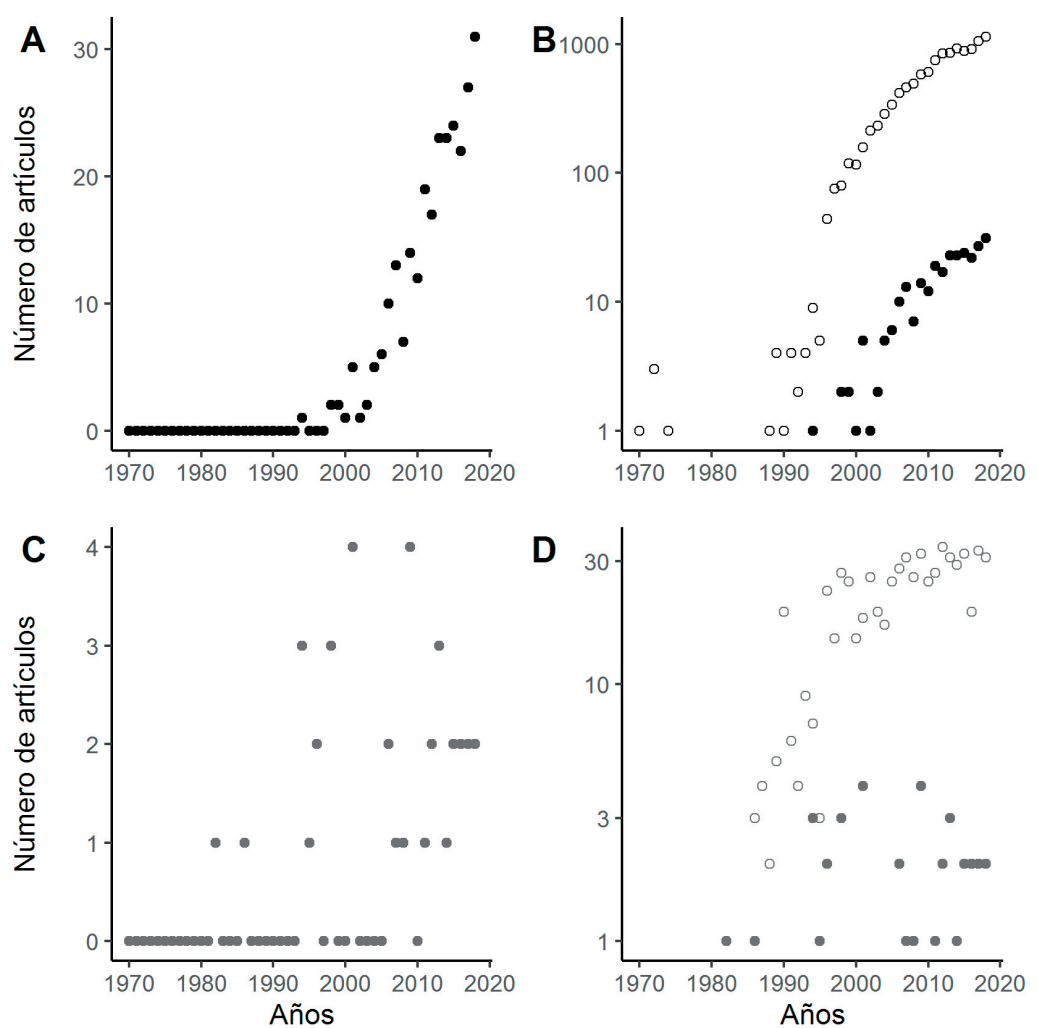

Figura 2. Número de artículos por año que incluyen las palabras A) macroecología o macroecology en artículos con al menos un autor argentino; B) macroecología o macroecology en artículos internacionales (círculos vacíos) y con al menos un autor argentino (círculos llenos); C) are(er)ografía o are(er)ography en artículos con al menos un autor argentino; D) are(er)ografía o are(er)ography en artículos internacionales (círculos vacíos), en comparación con artículos con al menos un autor argentino (círculos llenos). Los gráficos B y D muestran los ejes en escala logarítmica.

Figure 2. Number of articles per year that include the words A) macroecología or macroecology in articles with at least one Argentinian author; B) macroecología or macroecology in international articles (empty circles) and with at least one argentinian author (full circles); C) are(er)ografía o are(er)ography in articles with at least one argentinian author; D) are(er)ografía o are(er)ography in international articles (empty circles), compared to articles with at least one argentinian author (full circles). Graphics B and D show the axes on a logarithmic scale. 


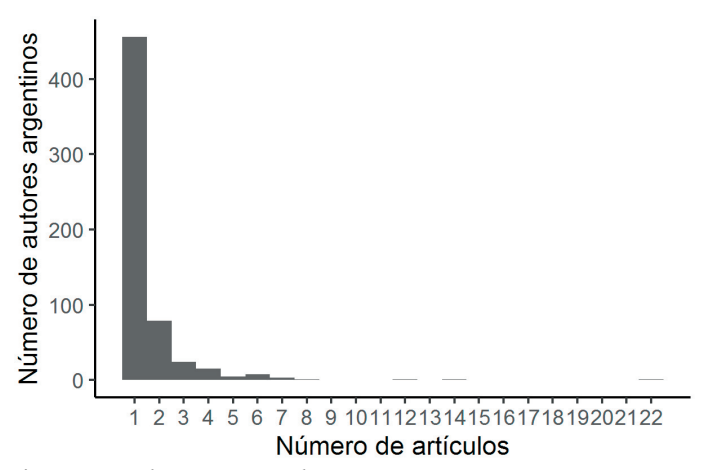

Figura 3. Número de artículos publicados por los autores argentinos conteniendo aproximaciones relacionadas con el enfoque macroecológico. La mayoría de los autores publicó un único artículo.

Figure 3. Number of articles published by Argentinian authors containing topics related to the macroecological approach. Most authors published a single article.

término incluido con mayor frecuencia como palabra clave en los trabajos fue Argentina $(\mathrm{n}=24)$, seguido por South America $(\mathrm{n}=19)$, species richness $(\mathrm{n}=16)$, Patagonia $(\mathrm{n}=15) \mathrm{y}$ macroecology $(\mathrm{n}=14)$ (Figura 4). El término areography o areografía fue incluido como palabra clave en un manuscrito.

El rango geográfico fue la variable más analizada en los trabajos de autores argentinos $(n=72)$, seguida por la riqueza de especies $(\mathrm{n}=68)$ y la diversidad funcional/análisis de rasgos $(n=42)$ (Figura 5A). Además, el conjunto de medidas de diversidad analizadas incluyó también a la abundancia, la diversidad beta y la diversidad genética o filogenética. Los estudios centrados en el tamaño corporal y en la diversidad genética o filogenética fueron menos frecuentes $(n=24$ y $n=20$, respectivamente). Los grupos más estudiados fueron los invertebrados, seguidos por las plantas y mamíferos mientras que los estudios macroecológicos basados en grupos de microorganismos, anfibios, peces y hongos fueron escasos en relación con el resto (Figura $5 B)$.

Enla mayoría delos 253 trabajos seleccionados $(n=182)$, el autor de correspondencia resultó de filiación argentina y la mayoría de estos trabajos fueron en coautoría con autores también argentinos o fueron publicados por un único autor argentino $(\mathrm{n}=122)$ (Figura 6). En el resto de los trabajos, las filiaciones más frecuentes del autor de correspondencia fueron Estados Unidos $(n=20)$, Brasil $(n=12)$, Reino Unido $(n=11)$ y España $(n=10)$. Las revistas en las que se publicaron con mayor frecuencia los artículos internacionales (i.e., Global Ecology and Biogeography, Journal of Biogeography y PLOS ONE) coincidieron con las revistas en las cuales se publicaron con mayor frecuencia los artículos

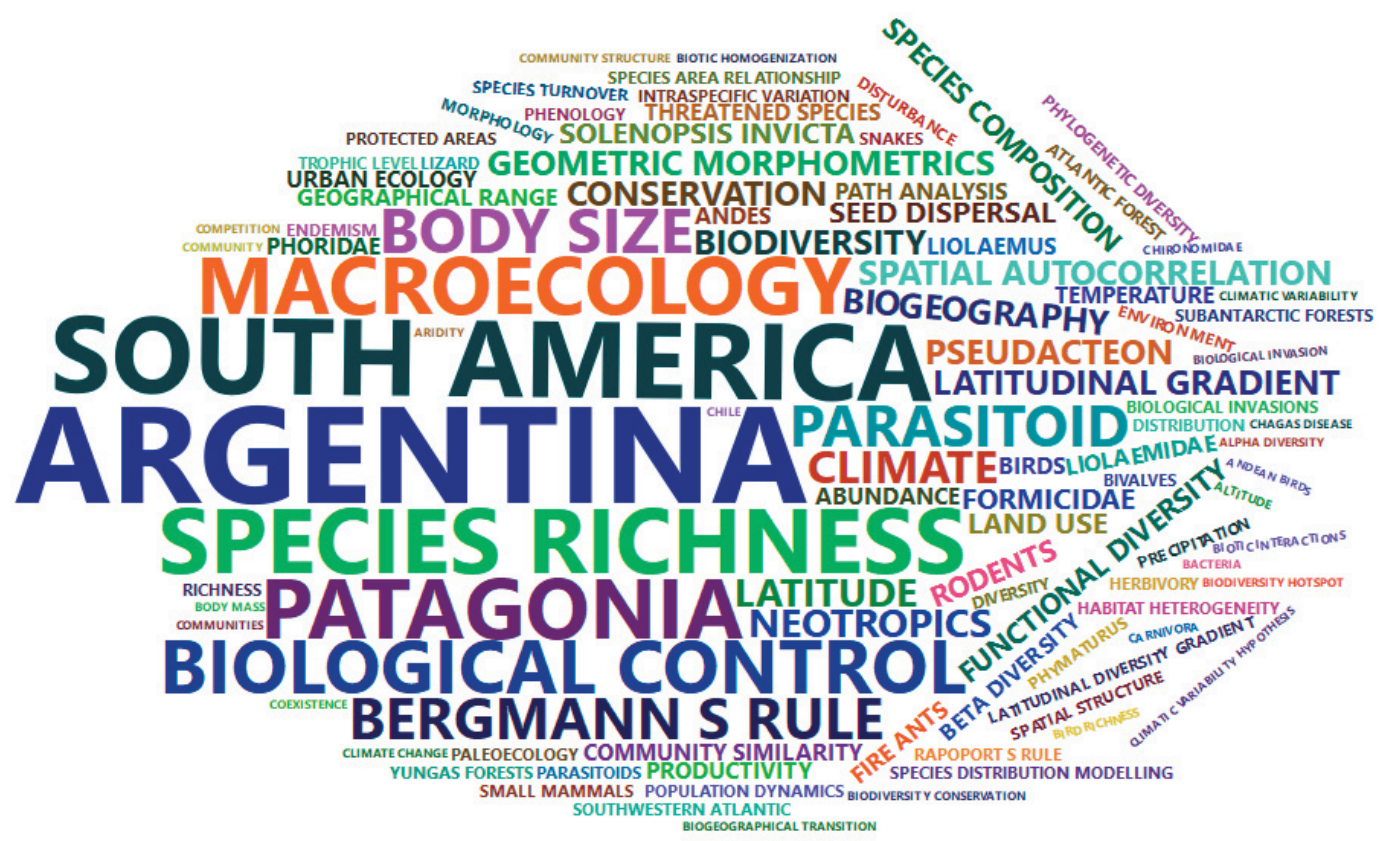

Figura 4. Nube de palabras clave más frecuentes incluidas en los 253 artículos argentinos con enfoque macroecológico. El tamaño indica la frecuencia relativa.

Figure 4. Cloud of most frequent keywords included in the 253 Argentinian articles with a macroecological approach. The size indicates the relative frequency. 

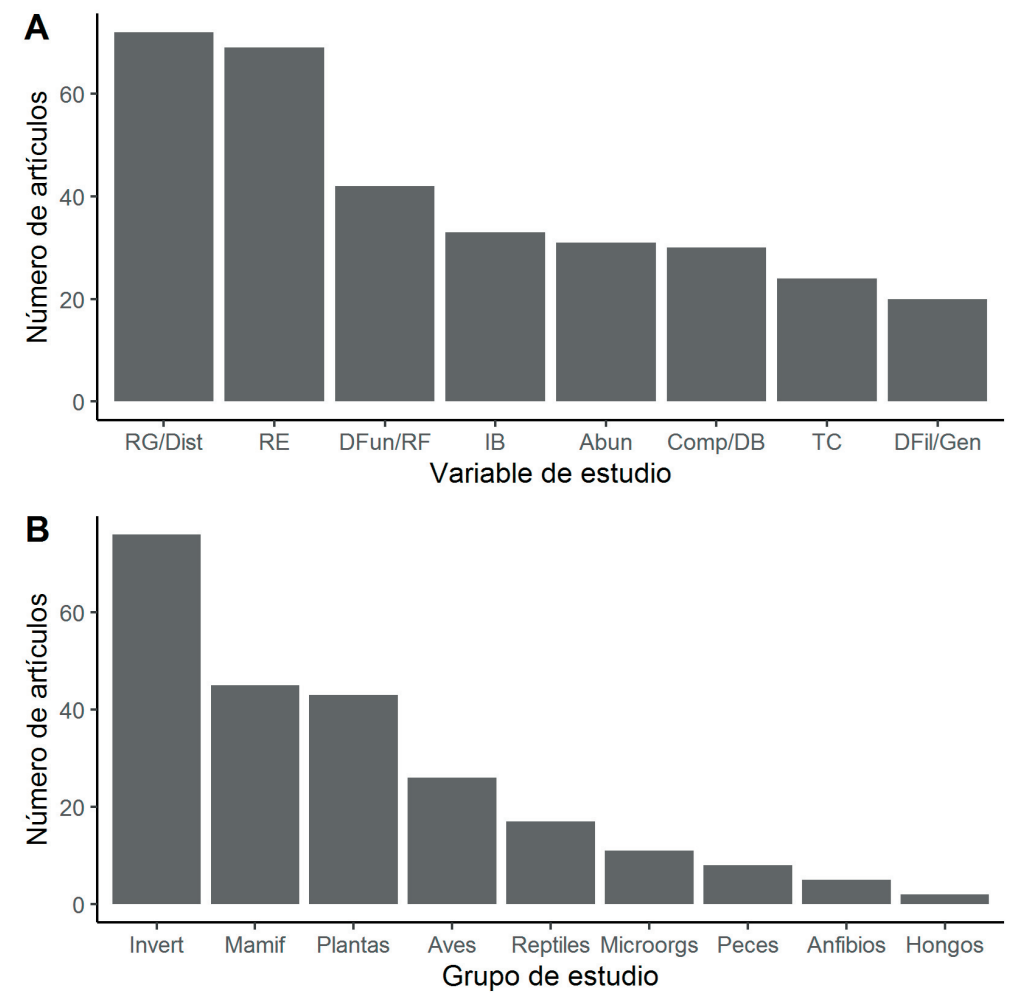

Figura 5. Distribución de frecuencias de A) las categorías de las variables estudiadas (RG/Dist: rango geográfico y Regla de Rapoport; RE: riqueza específica; DFun/RF: diversidad y rasgos funcionales; IB: interacciones biológicas; Abun: abundancia; Comp/DB: composición taxonómica y diversidad beta; TC: tamaño corporal y Regla de Bergmann; DFil/Gen: diversidad filogenética y genética); y B) grupos de estudio involucrados en los artículos macroecológicos con al menos un autor argentino (Invert: invertebrados; Mamif: mamíferos; Microorgs: microorganismos).

Figure 5. Frequency distribution of A) thematic areas (RG/Dist: geographical range and Rapoport's rule; RE: species richness; DFun/RF: diversity and functional traits; IB: biological interactions; Abun: abundance; Comp/DB: taxonomic composition and beta diversity; TC: body size and Bergmann's Rule; DFil/Gen: phylogenetic and genetic diversity); and B) study groups involved in macroecological articles with at least one Argentinian author (Invert: invertebrates; Mamif: mammals; Microorgs: microorganisms).

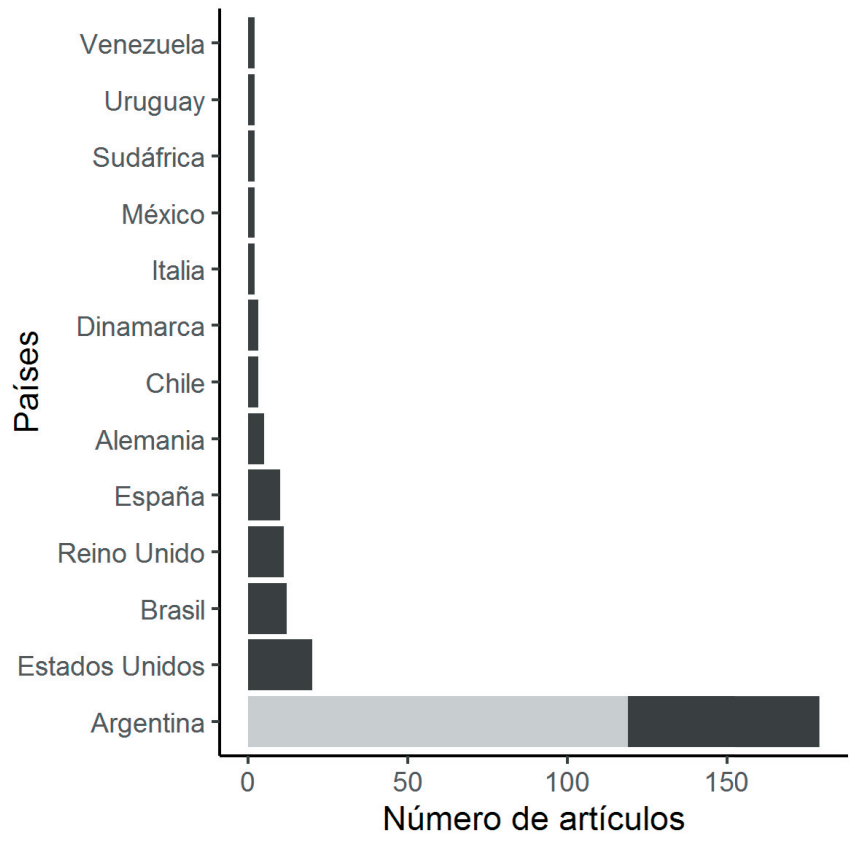

Figura 6. Número de artículos publicados por al menos un autor argentino, según el país de afiliación del autor de correspondencia. Se muestran los 13 países de afiliación de los autores de correspondencia que ocurren con mayor frecuencia. El resto de los países de afiliación ocurren como máximo una vez y no se incluyen en el gráfico. Dentro de los artículos cuyo autor de correspondencia es argentino se detalla la cantidad de artículos en los que todos los autores son argentinos (gris claro) o existen múltiples nacionalidades entre ellos (gris oscuro).

Figure 6. Number of articles published by at least one Argentinian author, according to the correspondence author's country of affiliation. The 13 countries of affiliation of the most frequent correspondence authors are shown. The rest of the countries of affiliation occur at most once and are not included in the graph. Among the articles whose correspondence author is Argentinian, the number of articles in which all the Argentinian authors are (light gray) or there are multiple nationalities among them (dark gray) is detailed. 
A

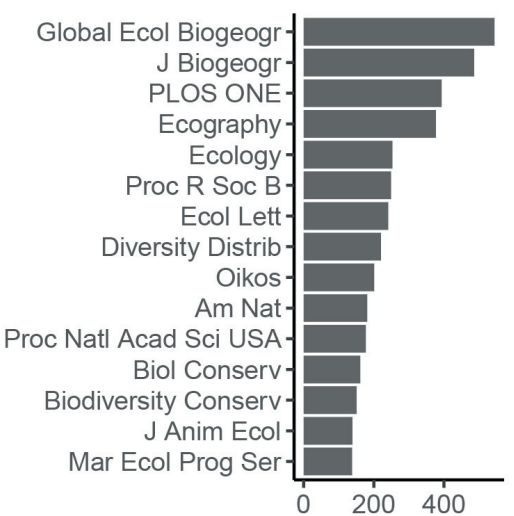

C

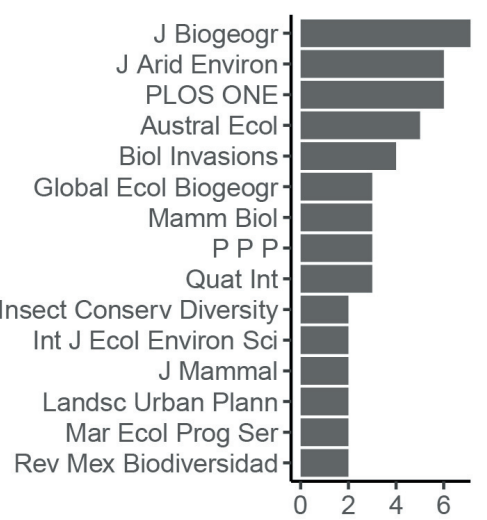

Número de artículos
B

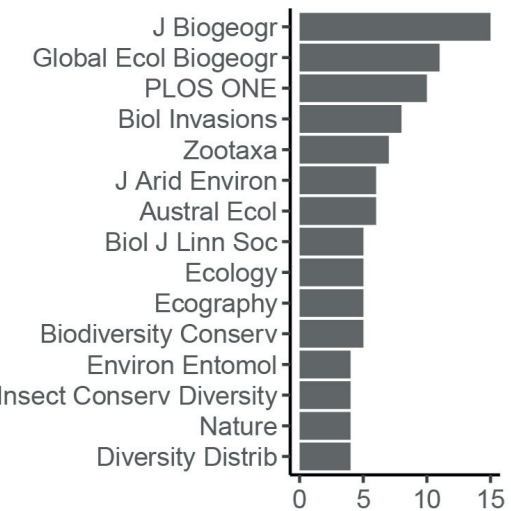

D

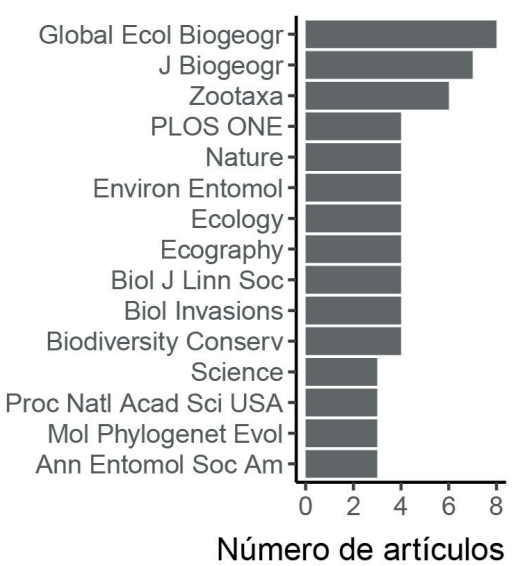

Figura 7. Revistas en las que principalmente se publican los estudios con enfoque macroecológico: a) internacionalmente; b) con al menos un autor argentino; que se desglosa en: c) únicamente autores argentinos y d) al menos un autor argentino y el resto de otras nacionalidades. Los nombres de las revistas se encuentran abreviados según la norma ISO4, a excepción de la revista Palaeogeography, Palaeoclimatology, Palaeoecology, que se abrevió como 'P P P’.

Figure 7. Journals in which studies with a macroecological focus are mainly published: a) internationally; b) with at least one Argentinian author, which is broken down into: c) only Argentinian authors and d) at least one Argentinian author and the rest of other nationalities. The names of the journals are abbreviated according to the ISO4 standard, with the exception of the magazine Palaeogeography, Palaeoclimatology, Palaeoecology, which was abbreviated as 'P P P'.

argentinos, aunque en otro orden (Figura 7A, B). Sin embargo, al analizar por separado las contribuciones de argentinos en coautoría con argentinos vs. argentinos en coautoría con extranjeros se observa que los argentinos en coautoría con argentinos publican con mayor frecuencia en Journal of Biogeography, Journal of Arid Environments y PLOS ONE (Figura 7C), y que los argentinos en coautoría con extranjeros publican más frecuentemente en Global Ecology and Biogeography, Journal of Biogeography y Zootaxa (Figura 7 D). Global Ecology and Biogeography es la revista en la que más publican los autores argentinos cuando están en coautoría con extranjeros (Figura 7 B-D).

Los artículos argentinos citaron con mayor frecuencia artículos y libros de autores no

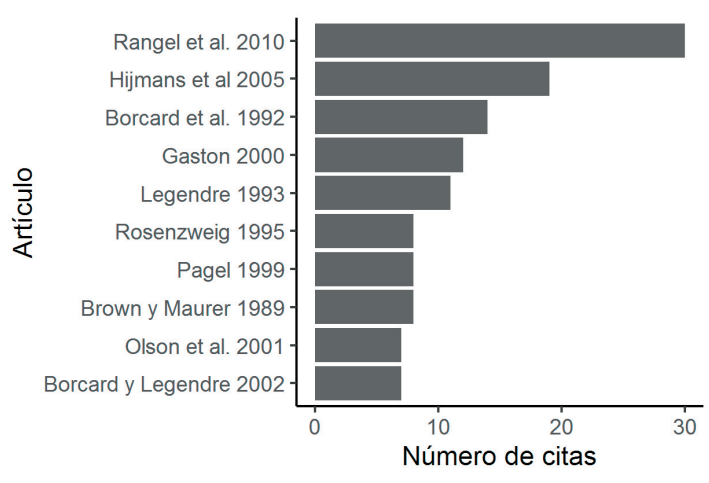

Figura 8. Distribución de frecuencias de los 10 artículos y libros citados principalmente en los artículos argentinos.

Figure 8. Frequency distribution of the 10 articles and books mainly cited in the Argentinian articles. 
argentinos (Figura 8). Los tres más citados fueron Rangel et al. 2010 (artículo relacionado con el software SAM, Spatial Analysis in Macroecology), Hijmans et al. (2005) (base global de datos climáticos) y Borcard et al. (1992) (artículo vinculado a la partición de la varianza en sus componentes espacial y ambiental).

\section{Discusión}

El recorrido de la ciencia argentina en su historia de contribuciones a la macroecología muestra un claro acompañamiento a su crecimiento como subdisciplina dentro de la ecología. Este acompañamiento se inició a mediados de los 90, casi dos décadas después de la aparición de la areografía de Rapoport (1975) y cinco años después de la consolidación de la macroecología de Brown y Maurer (1989). Tanto el momento del inicio de la tendencia creciente local del número de publicaciones como su relación con la tendencia global coinciden con lo encontrado en México (Rodríguez et al. 2017) y Brasil (de Moraes Weber 2018). Los autores argumentan que para México esto se podría explicar por la llegada al país de un número significativo de investigadores mexicanos luego de sus estadías postdoctorales en el exterior. En la Argentina, la tendencia se inicia con posterioridad al regreso de Rapoport a la Argentina luego del exilio en México, su establecimiento en Bariloche en 1984 y la formación de un grupo de investigación entre los años 1984 y 1989, fecha en que funda el laboratorio Ecotono (Rapoport 2015). A mediados de la década del noventa se publican los primeros trabajos con enfoque macroecológico por parte de los estudiantes de Rapoport (e.g., Farji-Brener and Ruggiero 1994; Ruggiero 1994). Hacia fines de la década del noventa, el número de trabajos que incorporaba una visión macroecológica se amplía, mostrando el inicio de una tendencia creciente que se explica por la consolidación como investigadores de esos estudiantes (e.g., Ruggiero et al. 1998; Monjeau et al. 1998), junto con los aportes de otros investigadores que utilizaron el enfoque (e.g., Cueto and Lopez de Casenave 1999; Ojeda et al. 2000). Sin embargo, considerando la totalidad de contribuciones de la comunidad científica nacional, los resultados muestran una baja cantidad de autores argentinos que publicó más de un artículo mencionando la macroecología o la areografía. Los resultados sugieren que la tendencia temporal creciente se debió en mayor medida a contribuciones eventuales que a programas regulares de investigación en la subdisciplina.

El desarrollo de la macroecología en la Argentina se relacionó con el regreso de Eduardo Rapoport al país, aunque esto no se reflejó en el número de menciones a la areografía que hacen los autores argentinos. El término areografía fue incluido como palabra clave en un único artículo argentino. Si bien el libro Areografía de Rapoport $(1975,1982)$ fue referencia en algunos trabajos argentinos y es frecuentemente citado en artículos extranjeros, la presentación de la macroecología (Brown and Maurer 1989) y su rápida consolidación dentro de la ecología (Brown 1995; Gaston and Blackburn 2000) posiblemente condujeron a que el término areografía haya caído en desuso y su definición no se haya adoptado como el títulodeun campodeinvestigaciónen símismo. Por otro lado, al considerarse en la actualidad a la areografía como parte de la biogeografía ecológica (Lomolino et al. 2017) es posible que el término también haya sido absorbido por el término biogeografía. En los artículos publicados, si bien el término macroecología apareció como una de las principales palabras clave, su frecuencia absoluta fue baja. Esto puede deberse a una cuestión idiosincrática y que simplemente no haya costumbre de hacer referencia a la subdisciplina (o el enfoque) del trabajo dentro de las palabras clave. También es posible que, dado que la biogeografía, la biogeografía ecológica, la ecología geográfica y la macroecología presentan límites difusos (Ruggiero 2001; Lomolino et al. 2017), se recaiga en el uso de términos relacionados con la biogeografía. En cualquier caso, la baja frecuencia en que la palabra macroecología se encuentra en títulos o resúmenes de los trabajos podría indicar que es necesario difundir y afianzar el enfoque, y hacerlo explícito en las palabras clave, para que los trabajos puedan ser encontrados en las búsquedas bibliográficas. Así, la inclusión del término macroecología con mayor frecuencia entre las palabras clave contribuiría a incrementar la valorización de la subdisciplina en el ámbito nacional e internacional.

La macroecología argentina se centró en el estudio de los rangos geográficos y en los patrones de distribución, y los invertebrados fueron los organismos focales principales. Por un lado, la frecuencia elevada en la que se abordan interrogantes relacionados con las áreas de distribución de las especies y los patrones relacionados con los rangos 
geográficos posiblemente responda a cómo se fue construyendo la historia de la macroecología en la Argentina y la influencia de Eduardo Rapoport. Los patrones de diversidad fueron también muy estudiados y se basaron sobre todo en la riqueza de especies, aunque en menor medida también se incorporaron la diversidad funcional y filogenética, aspectos de la diversidad que se consolidan como medidas clave (e.g., Cadotte et al. 2011; Faith 2002) que fueron incorporadas fuertemente en la macroecología internacional. Por otro lado, respecto de los grupos taxonómicos en estudio, el énfasis en el trabajo con invertebrados puede ser un reflejo de que en la Argentina se realiza un número sustancial de trabajos a escala local/regional donde no aplica el uso de grandes bases de datos de distribución disponibles en línea. Las bases de datos obtenidos de relevamientos a campo tienen el valor agregado de generar información de biodiversidad de lugares poco conocidos del sur de Sudamérica, una región que fue poco considerada para la generación de teoría. En general, los grupos taxonómicos más incorporados en los estudios argentinos fueron los mismos que se estudian a nivel mundial: aves, mamíferos, invertebrados y plantas. Sin embargo, tal como ocurriera en México, las frecuencias relativas no coincidieron porque los mamíferos fue el grupo más estudiado en México y las plantas lo fueron a nivel global (Rodríguez et al. 2017). Posiblemente, las historias particulares de los diferentes países determinan la preferencia por algún grupo sobre el resto en los estudios con enfoque macroecológico. Cabe señalar que en aéreas afines a la macroecología (e.g., la biogeografía y la filogeografía) se incluyen el uso de otros términos clave como corología. Las frecuencias relativas de los grupos taxonómicos en estas áreas afines pueden ser muy diferentes a lo encontrado para la macroecología, con un volumen alto de trabajos en plantas (datos no mostrados).

La Argentina contribuyó a la macroecología tanto con estudios desarrollados sólo por investigadores argentinos como con estudios producto de la interacción con investigadores de otras nacionalidades. Esa contribución se realizó con una frecuencia relativa elevada de artículos publicados en las principales revistas de macroecología en el ámbito internacional; los argentinos fueron autores de correspondencia en la mayoría de las publicaciones, lo cual muestra un rol protagónico. La interacción internacional se observó en mayor medida con Estados Unidos, Brasil, Gran Bretaña y España, países de trayectoria y gran contribución a la literatura macroecológica en general. En menor medida, se observaron publicaciones en revistas enfocadas en determinados sistemas o regiones cuando todos los autores son argentinos (e.g., Journal of Arid Environments, Austral Ecology), y en una revista centrada en la taxonomía (Zootaxa) o en una revista de alto impacto (Nature) cuando los coautores son extranjeros. Los resultados sugieren que los macroecólogos en la Argentina se centran en publicar en revistas de la especialidad con el objetivo de difundir conclusiones e implicancias relevantes para los ecólogos y biogeógrafos de todo el mundo (e.g., Journal of Biogeography y Global Ecology and Biogeography), pero que coexisten otras dinámicas de publicación. Así, la inserción de la Argentina en la macroecología conducida en el ámbito científico internacional es clara y se da en las principales revistas pertinentes; existe un amplio campo para su expansión y afianzamiento en el ámbito local.

En términos cuantitativos (i.e., cantidad de artículos), la contribución a la macroecología de los autores con filiación argentina se debió más a publicaciones eventuales de numerosos autores que a programas regulares de investigación en la subdisciplina. Si bien entre los autores que publican de forma regular en macroecología se encuentran quienes fueron estudiantes de Rapoport, la subdisciplina también fue llevada adelante por otros grupos de investigación. Las contribuciones de los programas regulares de investigación son muy relevantes en la literatura macroecológica (con artículos con más de 50 ó 100 citas internacionales; datos no mostrados). En términos cualitativos, el enfoque de la macroecología en Latinoamérica (y particularmente en la Argentina) permitió contribuir a comprender no sólo la regla de Rapoport (Rapoport 1975) y las características de la forma de las áreas (e.g., Ruggiero et al. 1998), sino también otras reglas ecogeográficas (e.g., Moreno et al. 2015), el gradiente de diversidad latitudinal (con más especies hacia los trópicos y menos hacia los polos) y su integración con la ecología y la historia evolutiva de las especies (e.g., Fergnani and Ruggiero 2017), los gradientes altitudinales (e.g., Werenkraut and Ruggiero 2011), la identificación de puntos calientes de diversidad (e.g., Ruggiero and Hawkins 2008) 
y áreas prioritarias de conservación (e.g., Nori et al. 2020), el estudio de la ecología de las especies invasoras (e.g., Folgarait et al. 2005) y vectores de enfermedades (e.g., Ceccarelli et al. 2019), o cómo el uso de la tierra influye sobre patrones espaciales de diversidad (e.g., Filloy et al. 2019), entre otros patrones y procesos.

El enfoque macroecológico ha sido adoptado de manera restringida en la Argentina; una de las posibles causas es la baja difusión local. Por ejemplo, hasta donde sabemos, en nuestro país no existen asignaturas de grado en macroecología, sino solamente cursos de postgrado eventuales. Otra posible causa es que, desde sus inicios, la macroecología fue criticada por analizar patrones estadísticos en un marco comparativo-correlativo en el que las hipótesis no son directamente puestas a prueba (Gaston and Blackburn 1999). Así, el contraste entre los factores potencialmente explicativos de un patrón se realiza a través del estudio simultáneo de múltiples hipótesis y comparando la fuerza de las asociaciones mediante el control estadístico de las variables predictoras. Este marco se debe a que, en general, no es posible realizar experimentos sobre grandes áreas geográficas o con un conjunto muy grande de especies (Brown 1995). Sin embargo, la macroecología puede servirse de 'experimentos naturales' dados por transformaciones bióticas o ambientales, tanto naturales como asociadas a las actividades humanas (Gaston and Blackburn 1999). Además, muchas veces para estos experimentos naturales es posible encontrar réplicas, como ocurre por ejemplo con montañas, islas o escenarios de uso de la tierra entre otros. Por otro lado, la macroecología invita también a imaginar, por ejemplo, a la diversidad de mamíferos del mundo como el resultado de un experimento natural de tantos posibles del cual no se cuenta con réplicas. Otro ejemplo son las áreas de distribución, que representan una construcción o modelo y la especie no está presente en cada $\mathrm{km}^{2}$ de su extensión. Con estilo, Rapoport (1975) explicó "las áreas geográficas de distribución son las sombras chinescas producidas por los taxones sobre la pantalla terrestre: es el medir, pesar y estudiar el comportamiento de fantasmas". El enfoque comparativo-correlativo no es una debilidad de la disciplina, sino que es una característica implícita que permitió generar un gran volumen de conocimiento.
La macroecología desarrollada por ecólogos argentinos es parte del legado de Eduardo Rapoport al sistema científico nacional y constituye una subdisciplina en crecimiento y expansión tanto en el ámbito nacional como internacional. No obstante, los resultados sugieren que los ecólogos argentinos incorporamos de forma parcial el enfoque macroecológico; son pocos los grupos de investigación centrados exclusivamente en este enfoque. En concordancia con el área temática fundacional de la macroecología por ecólogos argentinos (i.e., estudio de las áreas geográficas), en la Argentina existe un énfasis en estudios relacionados con áreas geográficas, patrones y rangos de distribución de especies. La macroecología desarrollada por ecólogos argentinos se encuentra inserta en el ámbito científico internacional, tanto por las colaboraciones con autores de otras nacionalidades como por la publicación de los artículos en revistas especializadas de alto impacto; en su mayoría, los autores argentinos asumen un rol protagónico en esos artículos. Aun así, la macroecología, si bien en vías de desarrollo, es un área de investigación relativamente vacante en la Argentina. Proponemos que una mayor asociación de la macroecología con otras subdisciplinas (e.g., la biología de la conservación), una mayor interacción internacional a nivel de proyectos (en los que la colaboración implique generar teoría) y una mayor difusión de los contenidos en asignaturas de grado puede contribuir al desarrollo de la macroecología en nuestro país. La Argentina posee uno de los gradientes ambientales más fuertes del mundo, involucrando el gradiente latitudinal y la cordillera de los Andes; la diversidad de ecorregiones es inmensa y las oportunidades de realizar experimentos naturales son únicas. Además, el libro de Rapoport contiene 12 capítulos y todos ellos constituyen una fuente vigente e inagotable de ideas; es muy posible que el legado continúe afianzándose en la Argentina.

Agradecimientos. M. I. Bellocq, A. Ruggiero y F. Villalobos nos impulsaron a avanzar en la elaboración del manuscrito. Las sugerencias y comentarios de A Ruggiero, la editora y un revisor nos permitieron mejorarlo. Este trabajo contó con el financiamiento de ANPCyTFONCYT, PICT 20163489 y PICT 2017 0843, UBACyT 2018 y UNCOMA 04/B228. 


\section{REFERENCIAS}

Aria, M., and C. Cuccurullo. 2017. bibliometrix: An R-tool for comprehensive science mapping analysis. Journal of Informetrics 11:959-975. https://doi.org/10.1016/j.joi.2017.08.007.

Blackburn, T. M., and K. J. Gaston. 2002. Macroecology is distinct from biogeography. Nature 418:723-723. https: //doi.org/10.1038/418723b.

Borcard, D., P. Legendre, and P. Drapeau. 1992. Partialling out the spatial component of ecological variation. Ecology 73:1045-1055. https://doi.org/10.2307/1940179.

Brown, J. H. 1984. On the relationship between abundance and distribution of species. The American Naturalist 124: 255-279. https://doi.org/10.1086/284267.

Brown, J. H. 1995. Macroecology. University of Chicago Press.

Brown, J. H. 1999. The legacy of Robert MacArthur: from geographical ecology to macroecology. Journal of Mammalogy 80:333-344. https://doi.org/10.2307/1383283.

Brown, J. H. 2017. Eduardo Hugo Rapoport (1927-2017): Argentina's special gift to ecology and biogeography. Mastozoología neotropical 24:269-271.

Brown, J. H. 2019. The genesis of macroecology: In memory of Brian Maurer. Global Ecology and Biogeography 28: 4-5. https://doi.org/10.1111/geb.12845.

Brown, J. H., and B. A. Maurer. 1989. Macroecology: the division of food and space among species on continents. Science 243:1145-1150. https://doi.org/10.1126/science.243.4895.1145.

Cadotte, M. W., K. Carscadden, and N. Mirotchnick. 2011. Beyond species: functional diversity and the maintenance of ecological processes and services. Journal of Applied Ecology 48:1079-1087. https://doi.org/10.1111/j.13652664.2011.02048.x

Ceccarelli, S., S. A. Justi, J. E. Rabinovich, J. A. F. Diniz Filho, and F. Villalobos. 2019. Phylogenetic structure of geographical co-occurrence among New World Triatominae species, vectors of Chagas disease. Journal of Biogeography 47(6): 1218-1231. https://doi.org/10.1111/jbi.13810.

Cueto, V. R., and J. L. de Casenave. 1999. Determinants of bird species richness: role of climate and vegetation structure at a regional scale. Journal of Biogeography 26:487-492. https://doi.org/10.1046/j.1365-2699.1999.00299.x.

de Moraes Weber, M. 2018. Panorama da macroecologia brasileira. Oecologia Australis 22:104-116.

Faith, D. P. 2002. Quantifying biodiversity: a phylogenetic perspective. Conservation Biology 16:248-252. https: //doi.org/10.4257/oeco.2018.2202.01.

Farji-Brener, A. G., and A. Ruggiero. 1994. Leaf-cutting ants (Atta and Acromyrmex) inhabiting Argentina: patterns in species richness and geographical range sizes. Journal of Biogeography 21:391-399. https://doi.org/10.2307/2845757.

Fergnani, P. N., and A. Ruggiero. 2017. The latitudinal diversity gradient in South American mammals revisited using a regional analysis approach: The importance of climate at extra-tropical latitudes and history towards the tropics. PLOS ONE 12:e0184057. https://doi.org/10.1371/journal.pone.0184057.

Filloy, J., G. A. Zurita, and M. I. Bellocq. 2019. Bird diversity in urban ecosystems: the role of the biome and land use along urbanization gradients. Ecosystems 22:213-227. https://doi.org/10.1007/s10021-018-0264-y.

Fisher, H. J. 2002. Macroecology: new, or biogeography revisited? Nature 417:787-787. https://doi.org/10.1038/ 417787c.

Folgarait, P. J., O. Bruzzone, S. D. Porter, M. A. Pesquero, and L. E. Gilbert. 2005. Biogeography and macroecology of phorid flies that attack fire ants in south-eastern Brazil and Argentina. Journal of Biogeography 32:353-367. https: //doi.org/10.1111/j.1365-2699.2004.01177.x

Gaston, K. J., and T. M. Blackburn. 1999. A critique for macroecology. Oikos 84:353-368. https://doi.org/10.2307/ 3546417.

Gaston, K. J., and T. M. Blackburn. 2000. Macroecology: pattern and process. Blackwell, Oxford. https://doi.org/10.1002/ 9780470999592.

Hawkins, B.A. 2001. Ecology's oldest pattern? Trends in Ecology and Evolution 16:470. https://doi.org/10.1016/S01695347(01)02197-8.

Hijmans, R. J., S. E. Cameron, J. L. Parra, P. G. Jones, and A. Jarvis. 2005. Very high-resolution interpolated climate surfaces for global land areas. International Journal of Climatology: A Journal of the Royal Meteorological Society 25(15):1965-1978. https://doi.org/10.1002/joc.1276.

Hoorn, C., J. M. Guayasamin, H. M. Ortega Andrade, and E. Bonaccorso. 2019. Celebrating Alexander von Humboldt's 250th anniversary: Exploring bio-and geodiversity in the Andes (IBS Quito 2019). Frontiers of Biogeography 11.2: e44178. https://doi.org/10.21425/F5FBG44178.

Humboldt, A. V., and A. Bonpland. 1807. Ideen zu einer Geographie der Pflanzen nebst einem Naturgemälde der Tropenländer. Tübingen, Germany.

Kent, M. 2005. Biogeography and macroecology. Progress in Physical Geography 29:256-264. https://doi.org/10.1191/ 0309133305pp447pr.

Lang, D., and G. Chien. 2018. Wordcloud2: Create Word Cloud by 'htmlwidget'. R package version 0.2.1. https: //CRAN.R-project.org/package=wordcloud2.

Lawton, J. H. 1999. Are there general laws in ecology? Oikos 84:177-192. https://doi.org/10.2307/3546712.

Lomolino, M. V., R. Riddle, and R. J. Whittaker. 2017. Pp. 730 in M. A. Sunderland (ed.). Biogeography. Fifth edition. Oxford University Press. 
Lomolino, M. V. 2020. Biogeography: A Very Short Introduction. Oxford University Press.

MacArthur, R. H., and E. O. Wilson. 1967. The theory of island biogeography. Princeton University Press. https: //doi.org/10.1093/actrade/9780198850069.001.0001.

MacArthur, R. H. 1972. Geographical ecology: patterns in the distribution of species. Princeton University Press.

Marquet, P. A. 2002. The search for general principles in ecology. Nature 418:723-723. https://doi.org/10.1038/ 418723c.

McGill, B. J. 2019. The what, how and why of doing macroecology. Global Ecology and Biogeography 28:6-17. https: //doi.org/10.1111/geb.12855.

Monjeau, J. A., E. C. Birney, K. Ghermandi, R. S. Sikes, L. Margutti, and C. J. Phillips. 1998. Plants, small mammals, and the hierarchical landscape classifications of Patagonia. Landscape Ecology 13:285-306. https://doi.org/10.1023/A: 1008012613305.

Moreno Azócar, D. L., M. G. Perotti, M. F. Bonino, J. A. Schulte, C. S. Abdala, and F. B. Cruz. 2015. Variation in body size and degree of melanism within a lizards clade: is it driven by latitudinal and climatic gradients? Journal of Zoology 295:243-253. https://doi.org/10.1111/jzo.12193.

Nori, J., R. Loyola, and F. Villalobos. 2020. Priority areas for conservation of and research focused on terrestrial vertebrates. Conservation Biology 34(5):1281-1291. https://doi.org/10.1111/cobi.13476.

O'Brien, E. 1998. Water-energy dynamics, climate, and prediction of woody plant species richness: an interim general model. Journal of Biogeography 25:379-398. https://doi.org/10.1046/j.1365-2699.1998.252166.x.

Ojeda, R. A., P. G. Blendinger, and R. Brandl. 2000. Mammals in South American drylands: faunal similarity and trophic structure. Global Ecology and Biogeography 9:115-123. https://doi.org/10.1046/j.1365-2699.2000.00167.x.

Outomuro, D., and F. Johansson. 2019. Wing morphology and migration status, but not body size, habitat or Rapoport's rule predict range size in North-American dragonflies (Odonata: Libellulidae). Ecography 42:309-320. https://doi.org/ 10.1111/ecog.03757.

R Core Team. 2019. R: A language and environment for statistical computing R Foundation for Statistical Computing, Vienna, Austria. URL: www.R-project.org.

Rabinovich, J. E., and E. H. Rapoport. 1975. Geographical variation of diversity in Argentine passerine birds. Journal of Biogeography 2:141-157. https://doi.org/10.2307/3037987.

Rangel, T. F., J. A. F. Diniz-Filho, and L. M. Bini. 2010. SAM: a comprehensive application for spatial analysis in macroecology. Ecography 33:46-50. https://doi.org/10.1111/j.1600-0587.2009.06299.x.

Rapoport, E. H. 1975. Areografía: estrategias geográficas de las especies. México, MX: Fondo de Cultura Económica.

Rapoport, E. H. 1982. Areography: geographical strategies of species. New York: Pergamon Press.

Rapoport, E. H. 2015. Aventuras y desventuras de un biólogo latinoamericano. FHN, Fundación de Historia Natural Félix de Azara.

Rapoport, E. H., E. Ezcurra, and B. Drausal. 1976. The distribution of plant diseases: a look into the biogeography of the future. Journal of Biogeography 3:365-372. https://doi.org/10.2307/3037978.

Rodríguez, P., F. Villalobos, A. Sánchez-Barradas, and M. E. Correa-Cano. 2017. La macroecología en México: historia, avances y perspectivas. Revista Mexicana de Biodiversidad 88:52-64. https://doi.org/10.1016/j.rmb.2017.10.006.

Ruggiero, A. 1994. Latitudinal correlates of the sizes of mammalian geographical ranges in South America. Journal of Biogeography 21:545-559. https://doi.org/10.2307/2845657.

Ruggiero, A., J. H. Lawton, and T. M. Blackburn. 1998. The geographic ranges of mammalian species in South America: spatial patterns in environmental resistance and anisotropy. Journal of Biogeography 25:1093-1103. https://doi.org/ 10.1046/j.1365-2699.1998.00253.x.

Ruggiero, A. 2001. Interacciones entre la biogeografía ecológica y la macroecología: aportes para comprender los patrones espaciales en la diversidad biológica. En J. L. Bousquets and J. J. Morrone (eds.). Introducción a la Biogeografía en Latinoamérica: Teorías, Conceptos, Métodos y Aplicaciones. Fac. Ciencias, UNAM, México, D.F.

Ruggiero, A., and B. A. Hawkins. 2008. Why do mountains support so many species of birds? Ecography 31:306-315. https://doi.org/10.1111/j.0906-7590.2008.05333.x.

Ruggiero, A., J. H. Lawton, and T. M. Blackburn. 1998. The geographic ranges of mammalian species in South America: spatial patterns in environmental resistance and anisotropy. Journal of Biogeography 25:1093-1103. https://doi.org/ 10.1046/j.1365-2699.1998.00253.x.

Schrodt, F., M. J. Santos, J. J. Bailey, and R. Field. 2019. Challenges and opportunities for biogeography - What can we still learn from von Humboldt? Journal of Biogeography 461631-1642. https://doi.org/10.1111/jbi.13616.

Stevens, G. C. 1989. The latitudinal gradient in geographical range: how so many species coexist in the tropics. The American Naturalist 133:240-256. https://doi.org/10.1086/284913.

Stevens, G. C. 1992. The elevational gradient in altitudinal range: an extension of Rapoport's latitudinal rule to altitude. The American Naturalist 140:893-911. https://doi.org/10.1086/285447.

Villalobos, F., A. Lira-Noriega, and P. Rodríguez. 2018. Is species geographic overlap constant across latitude? A homage to EH Rapoport. Oecologia Australis 22:144-155. https://doi.org/10.4257/oeco.2018.2202.04.

Werenkraut, V., and A. Ruggiero. 2011. Quality of basic data and method to identify shape affect richness-altitude relationships in meta-analysis. Ecology 92:253-260. https://doi.org/10.1890/09-2405.1. 